\title{
Exposición fetal a glucocorticoides se asocia al desarrollo cerebral en preadolescentes (1)
}

\author{
Davis EP, Sandman CA, Buss C, Wing DA, Head K. Fetal glucocorticoid exposure is \\ associated with preadolescent brain development. Biol Psychiatry 2013.doi:pii:S0006- \\ 3223(13)00262-X. 10.1016/j.biopsych.2013.03.009.
}

Análisis crítico: Du Y. Hwang, Claudio Vera PG. MSc, Jorge Carvajal C. PhD.

Unidad de Medicina Materno-Fetal. División de Obstetricia y Ginecología. Facultad de Medicina. Pontificia Universidad Católica de Chile

\section{RESUMEN (1)}

Antecedentes: Los glucocorticoides juegan un papel crítico en la regulación normativa de desarrollo del cerebro fetal. La exposición a niveles excesivos puede tener consecuencias perjudiciales e interrumpir procesos madurativos. Esto puede ser especialmente cierto cuando los glucocorticoides sintéticos se administran durante el período fetal, como lo son para las mujeres en trabajo de parto prematuro. Este estudio investigó las consecuencias para el desarrollo del cerebro y los problemas afectivos de la exposición fetal a los glucocorticoides sintéticos. Métodos: El desarrollo del cerebro y los problemas afectivos fueron evaluados en 54 niños (56\% mujeres) de 6 a 10 años, que nacieron de término. Los niños fueron reclutados en dos grupos: aquellos con y sin exposición fetal a los glucocorticoides sintéticos. Se efectuaron imágenes de resonancia magnética estructural y se determinó el grosor cortical. Los problemas afectivos infantiles se evaluaron utilizando el Child Behavior Check List. Resultados: Los niños en el grupo de exposición fetal a glucocorticoides mostraron un adelgazamiento cortical bilateral significativo. Las mayores diferencias entre los grupos se encontraban en la corteza cingulada anterior rostral (rACC). Más de $30 \%$ de la rACC era más delgada entre los niños con la exposición de glucocorticoides fetal. Además, los niños con más problemas afectivos tenían un rACC más delgada a izquierda. Conclusiones: La exposición fetal a los glucocorticoides sintéticos tiene consecuencias neurológicas que persisten durante al menos 6 a 10 años. Los niños con exposición a glucocorticoides fetal tuvieron una corteza más delgada principalmente en el rACC. Nuestros datos indicando que el rACC se asocia con problemas afectivos, en conjunto con evidencia de que esta región está implicada en los trastornos afectivos, aumenta la posibilidad de que los cambios neurológicos asociados con glucocorticoides aumenten la vulnerabilidad a problemas de salud mental.

\section{ANÁLISIS DE LA INVESTIGACIÓN}

\section{A. Relevancia clínica de la investigación}

Los glucocorticoides sintéticos son usados ampliamente en la obstetricia actual para reducir la morbimortalidad en los recién nacidos de pretérmino. Los glucocorticoides más usados son la betametasona y la dexametasona. Estos fármacos son capaces de cruzar la barrera placentaria e influir directamente en el desarrollo del feto (2). Los efectos benéficos de los corticoides sobre la morbimortalidad fetal están bien avalados por sólida evidencia (2), pero se ha observado que los glucocorticoides también pueden afectar el desarrollo del cerebro fetal. Experimentos animales han demostrado que la exposición fetal a corticoides altera el neurodesarrollo en roedores, ovejas y primates (3-8). Estudios en humanos han asociado el uso de corticoides antenatales con disminución del volumen y complejidad de los pliegues corticales $(9,10)$. Las regiones límbicas y prefrontales serían las más afectadas por su abundancia de receptores para glucocorticoides (11). El objetivo 
de este estudio es determinar la influencia a largo plazo de la exposición fetal a los corticoides y determinar si los cambios corticales están asociados a problemas afectivos en preadolescentes de entre 6 y 10 años.

\section{B. El estudio (1)}

Diseño: Estudio observacional, de cohorte retrospectiva. Realizado por un equipo multidisciplinario de las Universidades de Denver y California Irvine, EEUU. Pacientes: 54 niños entre 6 y 10 años. Grupo exposición a corticoides: 18 niños que recibieron corticoides entre las 24 y 34 semanas de gestación; nacidos de término, de embarazos únicos, con peso adecuado a la edad gestacional y sin patologías. Grupo control: 36 niños de características similares. No hubo diferencias significativas en las características de las madres o el contexto socioeconómico. Eventos de interés: Grosor de la corteza cerebral. Problemas afectivos y conductuales de los niños. Asociación entre estos dos eventos. Métodos: Medición de grosor cortical por medio de resonancia magnética de cerebro, procesada por un software especial. Aplicación de cuestionarios validados por entrevistadores entrenados y supervisados por un psicólogo. Resultados: No hubo diferencia significativa en problemas afectivos. $4 \%$ de la corteza cerebral fue más delgada en el grupo expuesto a corticoides. El área cortical con mayor adelgazamiento cortical fue el rACC, con un $30 \%$ de su superficie más delgada, en un 8 y $9 \%$ (hemisferio izquierdo y derecho, respectivamente). El grosor del rACC del hemisferio izquierdo se asoció significativamente a problemas afectivos en el grupo comparación $(r=-0,45 ; p<0,01)$, no así en el grupo expuesto a corticoides $(r=-0,1 ; p=$ no significativo).

\section{Análisis crítico}

Estudio de diseño observacional que en general respeta la formalidad de un estudio de estas características. La introducción explica el contexto científico y las razones de la investigación. Los objetivos del trabajo están claramente expuestos. Se especifican los criterios de inclusión y exclusión, tomando en cuenta las variables que podrían interferir en los eventos de interés. Los grupos resultaron comparables. Los métodos para constatar los eventos están claramente descritos. Incluyen el uso de cuestionarios estandarizados y validados en la literatura. Se usó un programa computacional estandarizado para el análisis de la corteza cerebral por RM. El estudio presenta fallas en su metodología: no se describe el cálculo del tamaño muestral, siendo esto una omisión crítica, ya que el cálculo del tamaño muestral se relaciona directamente con la capacidad del estudio de apoyar la hipótesis propuesta, disminuyendo el error al azar. Todos los resultados obtenidos en este estudio pueden ser atribuibles al azar. En cuanto a los resultados, el grosor de la corteza cerebral por sí mismo no es un evento clínicamente significativo. Se describe una asociación entre grosor cortical y los problemas afectivos, pero ésta se observó sólo en el grupo comparación y no en la población expuesta. Este hallazgo no tiene ninguna relación con el objetivo del estudio y podría ser sólo una asociación atribuible al azar. Conclusiones. Los resultados de este estudio no son de utilidad en nuestra práctica, ya que tienen un alto riesgo de error al azar. Siendo los corticoides la principal terapia para disminuir la morbimortalidad perinatal en prematuros, la asociación de riesgo que se sugiere en este trabajo no justifica cambios en nuestra conducta clínica habitual.

\section{REFERENCIAS}

1. Davis EP, Sandman CA, Buss C, Wing DA, Head K. Fetal glucocorticoid exposure is associated with preadolescent brain development. Biol Psychiatry 2013. doi:pii: S0006-3223(13)00262-X. 10.1016/j.biopsych.2013.03.009.

2. Kajantie E, Raivio T, Janne OA, Hovi P, Dunkel L, Andersson S. Circulating glucocorticoid bioactivity in the preterm newborn after antenatal betamethasone treatment. J Clin Endocrinol Metab 2004;89:39994003.

3. Roberts D, Dalziel S. Antenatal corticosteroids for accelerating fetal lung maturation for women at risk of preterm birth. Cochrane Database Syst Rev 2006;(3):CD004454.

4. Seckl JR. Glucocorticoids, developmental "programming" and the risk of affective dysfunction. Prog Brain Res 2008;167:17-34.

5. Trejo JL, Cuchillo I, Machín C, Rúa C. Maternal adrenalectomy at the early onset of gestation impairs the postnatal development of the rat hippocampal formation: effects on cell numbers and differentiation, connectivity and calbindin-D28K immunoreactivity. J Neurosci Res 2000;62:644-67.

6. Welberg LA, Seckl J. Prenatal stress, glucocorticoids and the programming of the brain. J Neuroendocrinol 2001;13:113-28.

7. Weinstock M. Alterations induced by gestational stress in brain morphology and behaviour of the offspring. Prog Neurobiol 2001;65:427-51.

8. Korte C, Styne D, Merritt AT, Mayes D, Wertz A, Helbock HJ. Adrenocortical function in the very low birthweight infants: Improved testing for sensitivity and association with neonatal outcome. J Pediatr 1996;128:257-63.

9. Meaney MJ, Diorio J, Francis D, Widdowson J, La Plante $\mathrm{P}$, Caldui $\mathrm{C}$, et al. Early environmental regulation of for brain glucocorticoid gene expression: Implications for adrenocortical response to stress. Dev Neurosci 1996;18:49-72. 
10. Murphy BP, Inder TE, Huppi PS, Warfield S, Zientara GP, Kikinis R, et al. Impaired cerebral cortical gray matter growth after treatment with dexamethasone for neonatal chronic lung disease. Pediatrics 2001;107:217-21.
11. Modi N, Lewis H, Al-Naqeeb N, Ajayi-Obe M, Dore CJ, Rutherford M. The effects of repeated antenatal glucocorticoid therapy on the developing brain. Pediatr Res 2001;50:581-5. 